SYPHILIS

\title{
Community based syphilis screening: feasibility, acceptability, and effectiveness in case finding
}

\author{
N L Lambert, M Fisher, J Imrie, R Watson, C H Mercer, J V Parry, A Phillips, A Iversen, N Perry, \\ G L Dean
}

Sex Transm Infect 2005;81:213-216. doi: 10.1136/sti.2004.013144

See end of article for authors' affiliations

....................

Correspondence to: Dr Gillian Dean, Lawson Unit, Outpatient Building, Royal Sussex County Hospital, Eastern Road, Brighton BN2 5BE, UK; Gillian.Dean@bsuh.nhs.uk

Accepted for publication 26 January 2005

Objectives: To investigate the feasibility and acceptability of implementing community based syphilis screening using different sample collection techniques, and its effectiveness in screening at-risk populations and identifying new syphilis cases.

Methods: Two phases of syphilis screening were conducted in venues frequented by men who have sex with men (MSM). Phase 1 used venepuncture and phase 2 a validated saliva test. Evaluation used quantitative data from testers, venues and the local genitourinary medicine (GUM) clinic, and qualitative data from venue and programme staff.

Results: 1090 MSM were tested over 7 weeks. $62 \%$ of testers had not attended a GUM clinic in the past year. $64 \%$ of testers reported $\geqslant 2$ sexual contacts in the past 90 days and $11 \%$ reported $\geqslant 10$. Similar diagnosis rates were recorded for phase $1(1.4 \%)$ and phase $2(1.8 \%)$. There was greater uptake of testing with the saliva test in saunas during phase 2.

Conclusions: Syphilis screening in gay venues is feasible and acceptable to at-risk MSM, and reaches a group not routinely accessing GUM services. The low case detection for syphilis suggest this approach, while unlikely to contain outbreaks, may be more useful if combined with screening for other sexually transmitted infections and effective health promotion strategies.

A s part of its response to increasing rates of sexually transmitted infections (STI), the UK National Strategy for Sexual Health and HIV outlines key service development needs. These include improved service delivery to at-risk populations. ${ }^{1}$ One such approach has been the implementation, in commercial gay venues, of syphilis screening programmes for men who have sex with men (MSM). However, to date, the evidence supporting such innovative community interventions is largely anecdotal, with different programmes reporting varied success rates. ${ }^{2-4}$

Brighton has had an ongoing syphilis outbreak since 1999. In keeping with similar outbreaks among MSM, it is characterised by high rates of HIV co-infection $(40 \%$ of cases) and predominantly anonymous and/or untraceable sexual partners ( $86 \%$ of all reported contacts). In response to growing local concern and the outbreak characteristics, we implemented a community based syphilis screening programme and report the findings of our evaluation here.

\section{METHODS}

The screening programme was undertaken in two phases and involved a collaboration between a community based organisation (CBO), the local genitourinary medicine (GUM) clinic, the primary care trust (PCT), and research partners. Our overall approach was similar to screening programmes in Dublin and Manchester. ${ }^{2}{ }^{3}$ During each session, one to four CBO workers circulated venues explaining the testing procedure. GUM clinic staff (one or two) collected a specimen from customers in a designated area of the venue and collected basic clinical and evaluative data. Staff also recorded the type of venue, estimated population, and numbers testing during each session. Those testing positive were contacted within 15 days and invited to attend the GUM clinic for confirmatory testing, treatment, and contact tracing.
Phase 1 "Only a little prick"

Phase 1 ran for 4 weeks in autumn 2002 and used conventional venepuncture. All local gay venues were invited to participate to provide as comprehensive a service as possible, and to identify any associations between type of venue and infection rates. Venues were excluded only where managers/owners declined.

\section{Phase 2 "Suck it and see"}

The second phase of the screening programme ran for 3 weeks during summer 2003. The shorter recruitment period was in response to customer saturation observed during the last stage of the first programme. The selection of venues in phase 2 was service driven rather than answering a specific research question. Screening was concentrated in a smaller number of venues where data from phase 1 demonstrated that men screened reported more casual and anonymous sexual partners and were therefore potentially at greater risk of syphilis ${ }^{6}$ Testing in phase 2 involved a newly validated saliva assay rather than venepuncture.

The salivary test was devised and validated as part of a separate study organised by the Health Protection Agency (report in preparation). Tests on 167 subjects with serological evidence of infectious syphilis showed an overall sensitivity of $86 \%$, comprising $71 \%$ for primary, $93 \%$ for secondary, and $90 \%$ for early latent syphilis. Tests on 139 controls revealed two reproducible false positives. All cases identified during the screening phases were confirmed using standard serological testing.

\section{Evaluation design}

Our evaluation incorporated four key outcomes: acceptability and feasibility to customers and venues, effectiveness in

Abbreviations: $C B O$, community based organisation; $G U M$, genitourinary medicine; MSM, men who have sex with men; PCT, primary care trust; STI, sexually transmitted infections 
testing at-risk populations, effectiveness in identifying new syphilis cases, and the comparative acceptability and feasibility of using saliva samples versus conventional venepuncture. "At-risk" populations were defined as MSM similar to known syphilis cases in age and number of sexual partners, or MSM with no recent history of GUM attendance, despite multiple sexual partners. Qualitative interviews and focus groups were conducted with programme staff and venue managers and analysed to identify factors that impeded or facilitated "feasibility, acceptability, and effectiveness in screening at-risk populations".

\section{RESULTS}

Feasibility and acceptability to customers and venues In phase 1 , all 30 local gay venues were invited to participate and $23(77 \%)$ agreed. In phase 2's more focused screening, 16 venues, including 13 from the previous phase and three newly opened ones, were approached and all agreed to participate. In total, 588 MSM were tested during phase 1 and 502 during phase 2 . The proportion of the venue population tested varied between sessions, between venue types, and between phases of the screening programme. Lowest uptake rates were in cruising grounds and the highest in saunas (table 1).

Qualitative data identified the following facilitators of acceptability: the strong relation between the CBO and local venues, provision of a separate testing area, and involving venue staff in promoting the programme (wearing campaign T-shirts and testing themselves). Barriers to acceptability included testing during "happy hours" and sessions provided by all female clinic staff.

\section{Effectiveness in testing at-risk populations}

Overall, 62\% (672/1090) of testers had not been to a GUM clinic in the past year. Qualitative findings suggested the screening programme acted as a valuable health promotion tool, raising awareness and encouraging some groups-for example, some sauna users who did not access other areas of the gay scene, to attend GUM services. Of those who had not recently attended GUM services, 65\% (434/672) reported $\geqslant 2$ sexual partners in the past 90 days. In both phases testers were similar to early syphilis cases already diagnosed in the GUM clinic with respect to age and number of partners in the past 90 days (table 1). Comparison of testers recruited from different venue types showed that in both phases, public sex site testers were significantly older $(\mathrm{p}<0.001)$, and reported significantly more partners in the past 90 days $(\mathrm{p}<0.001)$.

\section{Effectiveness in identifying new index cases of syphilis}

From 1090 tests a total of 17 cases of previously undiagnosed syphilis were detected, giving an overall new diagnosis rate of $1.6 \%$. Of these 17 cases, six were confirmed as early syphilis. The remaining 11 had either not attended GUM services in the past year, or not had previous syphilis serology and were therefore classified as late latent syphilis. Case identification rates were similar in phase $1(1.4 \%)$ and phase $2(1.8 \%)$ (table 1). In the GUM clinic during phase 1 and phase 2, nine and seven early infectious syphilis cases were diagnosed, respectively, giving a diagnosis rate of $4 \%$ (16/397) in the clinical setting.

\section{Acceptability and feasibility of saliva samples versus venepuncture}

Testing uptake was higher in phase 2 in saunas, but did not differ between phases with any of the other venue types (table 1). The lower specificity of the saliva test resulted in a higher rate of false positives than occurred in phase 1. Qualitatively, saliva testing was more acceptable and easier to administer, although outreach workers reported customer

Table 1 Feasibility and acceptability based on venue and tester uptake in phases 1 and 2 of community syphilis screening

\begin{tabular}{|c|c|c|c|c|c|c|}
\hline & & \multicolumn{3}{|c|}{$\begin{array}{l}\text { Phase } 1 \\
\text { (4 weeks) }\end{array}$} & \multicolumn{2}{|c|}{$\begin{array}{l}\text { Phase } 2 \\
\text { (3 weeks) }\end{array}$} \\
\hline \multicolumn{7}{|l|}{ Feasibility } \\
\hline$\%$ of venues agreeing to participate & & \multirow{3}{*}{\multicolumn{3}{|c|}{$\begin{array}{l}77 \%(23 / 30) \\
70\end{array}$}} & \multicolumn{2}{|c|}{$100 \%(16 / 16)$} \\
\hline \multirow{2}{*}{\multicolumn{4}{|c|}{$\begin{array}{l}\text { Number of venue sessions } \\
\text { Acceptability }\end{array}$}} & & 57 & \\
\hline & & & & & & \\
\hline Total number of men testing & & \multicolumn{3}{|l|}{588} & \multicolumn{2}{|l|}{502} \\
\hline Overall \% of venue populations who tested & & \multicolumn{3}{|l|}{$9.8 \%$} & \multicolumn{2}{|l|}{$11.4 \%$} \\
\hline$\%$ of non-public sex site bar/club populations testing & & \multicolumn{3}{|l|}{$9.0 \%$} & \multicolumn{2}{|l|}{$9.3 \%$} \\
\hline$\%$ of sauna populations testing & & \multicolumn{3}{|l|}{$23.1 \%$} & \multicolumn{2}{|l|}{$34.8 \%$} \\
\hline$\%$ of cruising ground populations testing & & \multicolumn{3}{|l|}{$11.7 \%$} & \multicolumn{2}{|l|}{$11.4 \%$} \\
\hline$\%$ of sex on premises club populations testing & & \multicolumn{3}{|l|}{$12.0 \%$} & \multicolumn{2}{|l|}{$19.5 \%$} \\
\hline GUM non-attendance in last year & & \multirow{2}{*}{\multicolumn{3}{|c|}{$65 \%(n=380)$}} & \multirow{2}{*}{\multicolumn{2}{|c|}{$58 \%(n=292)$}} \\
\hline \multicolumn{2}{|l|}{ No of sexual contacts in past 90 days among all testers: } & & & & & \\
\hline 0-1 & & \multicolumn{3}{|c|}{$31 \%(n=181)$} & \multicolumn{2}{|c|}{$29 \%(n=144)$} \\
\hline $2-19$ & & \multirow{2}{*}{\multicolumn{3}{|c|}{$55 \%(n=325)$}} & \multirow{2}{*}{\multicolumn{2}{|c|}{$56 \%(n=283)$}} \\
\hline $20+$ & & \multirow{2}{*}{\multicolumn{3}{|c|}{$14 \%(n=82)$}} & & $15 \%(n=75)$ \\
\hline \multicolumn{4}{|l|}{ No of sexual contacts in last 90 days among non-attendees: } & & & \\
\hline 0-1 & & \multirow{2}{*}{\multicolumn{3}{|c|}{$\begin{array}{l}37 \%(n=139) \\
53 \%(n=202)\end{array}$}} & \multicolumn{2}{|c|}{$34 \%(n=99)$} \\
\hline $2-19$ & & & & & $53 \%(n=$ & \\
\hline $20+$ & & $10 \%(n=$ & & & $13 \%(n=$ & \\
\hline Difference between community testers and known syphilis cases: & $\begin{array}{l}\text { Phase } 1 \\
\text { testers } \\
\text { ( } \mathrm{n}=588 \text { ) }\end{array}$ & $\begin{array}{l}\text { Known } \\
\text { cases } \\
(n=93)\end{array}$ & p Value* & $\begin{array}{l}\text { Phase } 2 \\
\text { testers } \\
\text { (n=502) }\end{array}$ & $\begin{array}{l}\text { Known } \\
\text { cases } \\
\text { ( } n=156)\end{array}$ & p Value* \\
\hline Age (median) & 33 & 33 & 0.545 & 36 & 34 & 0.170 \\
\hline Number of sexual contacts in last 90 days (median) & 4 & 3 & 0.733 & 3 & 3 & 0.675 \\
\hline Effectiveness in identifying new index cases of syphilis & & & & & & \\
\hline No of EIA positive results & & 29 & & & 43 & \\
\hline No of previously undiagnosed syphilis cases & & 8 & & & 9 & \\
\hline No of previously undiagnosed syphilis cases that were early syphilis & & 4 & & & 2 & \\
\hline Co-infection & $1 \times \mathrm{HIV}+1$ & dy diagno & & $1 \times \mathrm{HIV}+1$ & ready diag & \\
\hline & $1 \times$ rectal & rhoea lcc & losed) & $1 \times$ rectal & norrhoea & agnosed) \\
\hline
\end{tabular}




\section{Key messages}

- Screening for syphilis in gay venues is feasible and acceptable to at-risk MSM, and reaches a group not routinely accessing GUM services

- A strong collaborative relationship between local healthcare providers, venue owners, and community based organisations is key to the success of such programmes

- While saliva testing for syphilis has certain logistical advantages, these may be offset by reduced sensitivity/ specificity and the inability to distinguish new from old infection

- Although this approach is unlikely to contain syphilis outbreaks, health promotion gains should not be underestimated. Further exploration linking outreach work to screening for other STIs is justified

misconceptions about whether the test could be performed after drinking alcohol.

\section{DISCUSSION}

The highly infectious and frequently asymptomatic nature of early syphilis, coupled with high rates of HIV co-morbidity and anonymous sexual contacts, demand innovative and proactive syphilis case finding strategies. However, these strategies need to be evaluated before widespread implementation is undertaken. Our findings show that community screening is feasible and acceptable to MSM, the majority of whom had not attended GUM services in the last year. Saliva testing increased uptake in saunas and an overall diagnosis rate of $1.6 \%$ was achieved. However the public health significance of this case finding activity is less clear as most new diagnoses were not categorised as "infectious syphilis."

Diagnosis rates were similar to those in Manchester's gay venues in 2000-1 (1.4\%), ${ }^{3}$ and somewhat higher than in Los Angeles' gay venues in $1999(0.48 \%) .{ }^{4}$ By contrast, the diagnosis rate in Dublin gay venues in 2000-1 was considerably higher $(7.5 \%){ }^{2}$ The number of uncontrolled variables in a programme evaluation compared to an experimental study makes these differences hard to interpret and anonymous prevalence surveys may be the most useful way to investigate such variation.

Saliva testing was easier to administer, and demanded fewer clinical staff and health and safety requirements than venepuncture. Because the test does not differentiate between old and new infection, we would advise avoiding testing those with a previous history of syphilis. However, it does allow teams to go into a venue, screen large numbers, identify the small proportion of positive individuals, and fast track them for a clinic appointment and full laboratory investigation for all infections. Overall, saliva testing did not increase testing uptake, although it did increase uptake in saunas. It is also possible that saliva testing led to increased overall uptake among MSM at lower risk of syphilis, based on the higher proportion of testers in phase 2 that reported GUM attendance in the previous year compared to phase 1 .

The value of undertaking a community based syphilis screening in outbreak management depends on the populations, the testing venues, and the rationale for its implementation. Our findings concur with reports from other community based screening programmes that this approach results in low case detection rates. ${ }^{4-10}$ It is unlikely therefore that such interventions will have a significant impact on outbreak evolution. In comparison, testing for other STIs in community settings, particularly male only saunas, has proved more fruitful with prevalence rates of $10.7 \%$ for chlamydia and/or gonorrhoea, ${ }^{11}$ and $11.5 \%$ for gonorrhoea alone. ${ }^{12}$ As we have shown, focusing testing on such venues may identify individuals with higher numbers of partners, and therefore greater overall risk for STI acquisition.

In summary, we have shown it is possible to identify a large population of MSM, at risk of STIs, who are not routinely accessing GUM services, but who are willing to test in a community environment. We believe that such programmes in future should not restrict themselves to syphilis identification, but rather should be used to screen for multiple STIs and encourage further effective health promotion.

\section{ACKNOWLEDGEMENTS}

The authors wish to thank those who tested and who made their venues available to the project, the Department of Health for providing funding, and the staff of the Claude Nicol Centre and the Lawson Unit, Royal Sussex Country Hospital, Brighton, for their patience, commitment, and support of this study.

\section{CONTRIBUTORS}

NL managed the screening programme and data collection and wrote the first draft of the paper; NL and JI designed the evaluation and data collection tools; CM, NL, and JI guided and performed the quantitative analysis; NL, JI, and RW performed qualitative data collection and analysis; AP and RW supervised the day to day running of the programme and staffing; RW coordinated community support and participation for the Brighton Syphilis Outbreak Project; JP led the saliva test validation and supervised laboratory testing of them; AI and NP provided programme advice; MF and GD were the principal investigators on the Brighton Syphilis Outbreak Project and contributed to all stages of the project and paper writing; all of the authors contributed to the design and development of the programme, paper review and comments on successive drafts.

\section{Authors' affiliations}

N L Lambert, M Fisher, A Phillips, N Perry, G L Dean, HIV/GUM Research Department, Residence Block, Brighton General Hospital, Elm Grove, Brighton BN2 3EW, UK

J Imrie, C H Mercer, Centre for Sexual Health and HIV Research, Royal Free and University College Medical School, University College London, Mortimer Market Centre, off Capper Street, London WCIE 6AU, UK R Watson, Terrence Higgins Trust South, 61 Ship Street, Brighton BN1 $1 \mathrm{AE}, \mathrm{UK}$

J V Parry, Centre for Infections, Health Protection Agency, 61 Colindale Avenue, London NW9 5EQ, UK

A Iversen, Surrey and Sussex Health Protection Unit, Health Protection Agency, 36-38 Friars Walk, Lewes BN7 2PB, UK

Funding: UK Department of Health.

Conflict of interest: none.

Ethical approval: Ethical approval for the Brighton Syphilis Outbreak Project, including this evaluation, was obtained from the Brighton and Mid-Sussex Local Research Ethics Committee

\section{REFERENCES}

Department of Health. The national strategy for sexual health and HIV. London: Department of Health Publications, 2001.

2 Hopkins S, Lyons F, Mulcahy F, et al. The great pretender returns to Dublin, Ireland. Sex Transm Infect $2001 ; 77: 316-18$.

3 Clark P, Cook PA, Bellis MA, et al. Re-emerging syphilis in the north west (Oral presentation). Leicester, UK: Proceedings of the 6th National CHAPS Conference, 26-27 March, 2003.

4 Chen JL, Kodagoda D, Lawrence M, et al. Rapid public health interventions in response to an outbreak of syphilis in Los Angeles. Sex Transm Dis 2002;29:277-84.

5 Poulton M, Dean GL, Williams DI, et al. Surfing with spirochaetes: an ongoing syphilis outbreak in Brighton. Sex Transm Infect 2001;77:319-21.

6 Imrie J, Lambert N, Phillips A, et al. Demographics, sexual partnerships and behaviour, and attitudes associated with recent syphilis diagnosis among men who have sex with men (MSM) on England's South Coast (Abstract number P97). Bath, UK: Proceedings of the BASHH/ASTDA Spring Meeting, 2004, 19-21 May. 
7 Debattista J, Dwyer J, Orth D, et al. Community screening for Neisseria gonorrhoeae and Chlamydia trachomatis among patrons of sex-on-premises venues: two years later. Venereology 2000;13:105-9.

8 Kahn RH, Scholl DT, Shane SM, et al. Screening for syphilis in arrestees: usefulness for community-wide syphilis surveillance and control. Sex Transm Dis 2002;29:150-6

9 Khan RA, Moseley KE, Thilges JN, et al. Community-based screening and treatment for STDs: results from a mobile clinic initiative. Sex Transm Dis 2003;30:654-8.
10 Morton AN, Wakefield T, Tabrizi SN, et al. An outreach programme for sexually transmitted infection screening in street sex workers using selfadministered samples. Int J STD AIDS 1999;10:741-7.

11 Lister NA, Smith A, Tabrizi S, et al. Screening for Neisseria gonorrhoeae and Chlamydia trachomatis in men who have sex with men at male-only saunas. Sex Transm Dis 2003;30:886-9.

12 Judson FN, Miller KG, Schaffnit TR. Screening for gonorrhea and syphilis in the gay baths-Denver, Colorado. Am J Public Health 1977;67:740-2.

\section{Clinical Evidence-Call for contributors}

Clinical Evidence is a regularly updated evidence-based journal available worldwide both as a paper version and on the internet. Clinical Evidence needs to recruit a number of new contributors. Contributors are healthcare professionals or epidemiologists with experience in evidence-based medicine and the ability to write in a concise and structured way.

Areas for which we are currently seeking authors:

- Child health: nocturnal enuresis

- Eye disorders: bacterial conjunctivitis

- Male health: prostate cancer (metastatic)

- Women's health: pre-menstrual syndrome; pyelonephritis in non-pregnant women

However, we are always looking for others, so do not let this list discourage you.

Being a contributor involves:

- Selecting from a validated, screened search (performed by in-house Information Specialists) epidemiologically sound studies for inclusion.

- Documenting your decisions about which studies to include on an inclusion and exclusion form, which we keep on file.

- Writing the text to a highly structured template (about 1500-3000 words), using evidence from the final studies chosen, within 8-10 weeks of receiving the literature search.

- Working with Clinical Evidence editors to ensure that the final text meets epidemiological and style standards.

- Updating the text every six months using any new, sound evidence that becomes available. The Clinical Evidence in-house team will conduct the searches for contributors; your task is simply to filter out high quality studies and incorporate them in the existing text.

- To expand the topic to include a new question about once every 12-18 months.

If you would like to become a contributor for Clinical Evidence or require more information about what this involves please send your contact details and a copy of your CV, clearly stating the clinical area you are interested in, to Klara Brunnhuber (kbrunnhuber@ bmigroup.com).

\section{Call for peer reviewers}

Clinical Evidence also needs to recruit a number of new peer reviewers specifically with an interest in the clinical areas stated above, and also others related to general practice. Peer reviewers are healthcare professionals or epidemiologists with experience in evidence-based medicine. As a peer reviewer you would be asked for your views on the clinical relevance, validity, and accessibility of specific topics within the journal, and their usefulness to the intended audience (international generalists and healthcare professionals, possibly with limited statistical knowledge). Topics are usually 1500-3000 words in length and we would ask you to review between $2-5$ topics per year. The peer review process takes place throughout the year, and our turnaround time for each review is ideally 10-14 days.

If you are interested in becoming a peer reviewer for Clinical Evidence, please complete the peer review questionnaire at www.clinicalevidence.com or contact Klara Brunnhuber (kbrunnhuber@bmigroup.com). 